\title{
The Schreier Refinement Theorem for Categories
}

\author{
By \\ Rudolf Fritsch and Oswald WyLer
}

We use the preceding note [1] to prove the following theorem.

Theorem. Any two normal series of subobjects of an object $G$ of a pointed category $\mathscr{C}$ have isomorphic refinements if $\mathscr{C}$ satisfies $\mathrm{Z} 2$, and the following condition.

$\mathrm{Z} 1 * . \mathscr{C}$ has cokernels of kernels, and kernels of any composition $e^{\prime} e$ of two cokernels e and $e^{\prime}$.

We note first that $\mathrm{Z} 1 *$ implies that every cokernel has a kernel, since every identity morphism is a cokernel of a zero morphism, and we have the following three lemmas.

Lemma $1 . \mathrm{Z1}^{*}$ is equivalent to the following statement: $\mathscr{C}$ has cokernels of kernels, and every possible pullback square

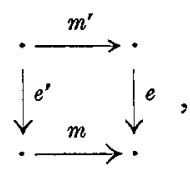

for a kernel $m$ and a cokernel $e$ with the same target, exists in $\mathscr{C}$.

Proof. If $\mathrm{Z}_{1}{ }^{*}$ is valid, let $e_{1}$ be a cokernel of $m$ and $m^{\prime}$ a kernel of $e_{1} e$. Then $e m^{\prime}=m e^{\prime}$ for a morphism $e^{\prime}$, and this obviously is the desired pullback. For the converse, we observe first that a cokernel of an identity morphism (which is a kernel of a zero morphism) produces a zero object. If $e: A \rightarrow B$ and $m=0: Z \rightarrow B$ for a zero object $Z$, then $m$ is a kernel of id $B$, and a pullback produces a kernel $m^{\prime}$ of $e$. Now if $e_{1} e$ is the composition of two cokernels and $m$ a kernel of $e$, then a pullback produces a kernel $m^{\prime}$ of $e_{1} e$.

Definition. A morphism $m$ of $\mathscr{C}$ will be called a subkernel if $m$ is the composition in $\mathscr{C}$ of kernels in $\mathscr{C}$, and $(A, a)$ will be called a subnormal subobject of $G$ if $a: A \rightarrow G$ is a subkernel in $\mathscr{C}$.

Lemma 2. (i) The intersection of any two subnormal subobjects $(A, a)$ and $(B, b)$ of an object $G$ of $\mathscr{C}$ exists in $\mathscr{C}$ and is a subnormal subobject of $\mathscr{C}$.

(ii) If $m m^{\prime}$ is defined in $\mathscr{C}$ and $m$ is a subkernel, then $m m^{\prime}$ is a subkernel if and only if $m^{\prime}$ is a subkernel. 
Proof. Let $a=a_{10} a_{20} \cdots a_{h 0}$ and $b=b_{01} b_{02} \cdots b_{0 k}$ for kernels $a_{i 0}$ and $b_{0 j}$. If $a_{i, j-1}$ and $b_{i-1, j}$ are defined and kernels, then a pullback

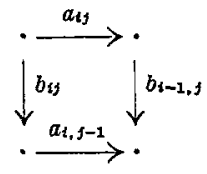

exists in $\mathscr{C}$, for kernels $a_{i j}$ and $b_{i j}$, by the dual of 2.3 in [1]. This defines kernels $a_{i j}$ and $b_{i j}$ recursively for $1 \leqq i \leqq h$ and $1 \leqq j \leqq k$. If $a^{\prime}=a_{1 k} a_{2 k} \cdots a_{h k}$ and $b^{\prime}=$ $=b_{h 1} b_{h 2} \cdots b_{h k}$, then $a^{\prime}$ and $b^{\prime}$ are subkernels, and the commutative square

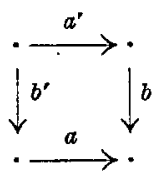

in $\mathscr{C}$ is the composition of $h \cdot k$ pullback squares, and hence a pullback. Thus $a \cap b$ exists and is subnormal.

The "if" part of (ii) is trivial. For the "only if" part, put $a=m m^{\prime}$ and $b=m$ in (i). Then we can carry out the construction of (i) if $m$ and $m m^{\prime}$ are subkernels, and we obtain $a^{\prime} \cong m^{\prime}$. Thus $m^{\prime}$ is a subkernel.

Lemma 3. If $f$ is a cokernel and $m$ a subkernel so that $f m$ is defined, then $f[m]$ and $f^{-1}[f[m]]$ exist in $\mathscr{C}$ and are subnormal, and $f\left[f^{-1}[f[m]]\right] \cong f[m]$ in $\mathscr{C}$.

Proof. Let $f: A \rightarrow B$, and let $m=a_{1} a_{2} \cdots a_{h}$ for kernels $a_{i}$. Put $m_{0}=$ id $A$ and $m_{i}=m_{i-1} a_{i}$ for $1 \leqq i \leqq h$, so that $m_{h}=m$. Define kernels $b_{i}$ and cokernels $e_{i}$ recursively in $\mathscr{C}$, using $\mathrm{Z} 2$, by putting $e_{0}=f$, and $e_{i-1} a_{i}=b_{i} e_{i}$ for $1 \leqq i \leqq h$. If we put $m_{0}^{\prime}=$ id $B$ and $m_{i}^{\prime}=m_{i-1}^{\prime} b_{i}$ for $1 \leqq i \leqq h$, then $m_{i}^{\prime} e_{i}=f m_{i}$, and $m_{i}^{\prime}$ is a subkernel. Thus $f\left[m_{i}\right] \cong m_{i}^{\prime}$, and $f[m] \cong m_{h}^{\prime}$ exists and is subnormal.

Now we construct recursively the following diagram

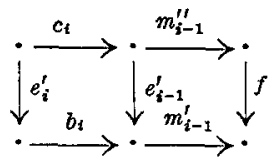

beginning with $e_{0}^{\prime}=f, m_{0}^{\prime}=$ id $B$ and $m_{0}^{\prime \prime}=$ id $A$. We assume that the righthand square is a pullback, with $m_{i-1}^{\prime \prime}$ a subkernel and $e_{i-1}^{\prime}$ a cokernel. This is satisfied for $i=1$. By Lemma 1 , the lefthand square can be constructed as a pullback, and then $c_{i}$ is a kernel, the rectangle is a pullback, and $m_{i}^{\prime \prime}=m_{i-1}^{\prime \prime} c_{i}$ defines a subkernel $m_{i}^{\prime \prime}$. Thus the construction can go on if $e_{i}^{\prime}$ is a cokernel. To see that $e_{i}^{\prime}$ is ar cokernel, put $b_{i} e_{i}^{\prime}=e_{i-1}^{\prime} c_{i}=b_{i}^{\prime} e_{i}^{\prime \prime}$ for a cokernel $e_{i}^{\prime \prime}$ and a kernel $b_{i}^{\prime}$, using Z2. Then $b_{i}^{\prime} \leqq b_{i}$ since $\left(e_{i}^{\prime \prime}, b_{i}^{\prime}\right)$ is an image. On the other hand, $f m_{i}=m_{i}^{\prime} e_{i}$, and thus $m_{i}=m_{i}^{\prime \prime} u_{i}$, $e_{i}=e_{i}^{\prime} u_{i}$, for a morphism $u_{i}$, and $b_{i} e_{i}=b_{i}^{\prime} e_{i}^{\prime \prime} u_{i}$ follows. But then $b_{i} \leqq b_{i}^{\prime}$ since $\left(e_{i}, b_{i}\right)$ is an image. Thus $b_{i}^{\prime}=b_{i} x, e_{i}^{\prime}=x e_{i}^{\prime \prime}$, for an isomorphism $x$, and $e_{i}^{\prime}$ is a cokernel. 
Now $f^{-1}\left[m_{i}^{\prime}\right] \cong m_{i}^{\prime \prime}$, and $f\left[m_{i}^{\prime \prime}\right] \cong m_{i}^{\prime}$, and the lemma is proved.

All results of [1] now become available, except for 4.2 , if we restrict ourselves to subnormal subobjects. The last part of Lemma 3 replaces 4.2 in proofs in [1]; no other changes are needed. Subobjects occuring in a normal series are ipso facto subnormal. Thus the Zassenhaus Lemma is valid for these subobjects, and the usual proof of the Schreier Theorem goes through without any changes.

\section{Reference}

[1] O. WyLer, The Zassenhaus Lemma for categories. Arch. Math. 22, 561-569 (1971).

Eingegangen am 21.6.1971

Anschrift der Autoren:

Rudolf Fritsch

Fachbereich Mathematik der Universität

Oswald Wyler

775 Konstanz

Department of Mathematics

Jacob-Burckhardt-Str.

Carnegie-Mellon-University

Pittsburgh, Pennsylvania 15213, USA 